\title{
EE-MAC: Energy Efficient Sensor MAC layer Protocol
}

\author{
Afraa Attiah*, Mustafa İlhan Akbaş, Mainak Chatterjee, Damla Turgut \\ Department of Electrical Engineering and Computer Science \\ University of Central Florida, Orlando, FL \\ Email: *afraa.attiah@knights.ucf.edu, \\ \{miakbas,mainak,turgut\}@eecs.ucf.edu
}

\begin{abstract}
Energy efficiency is of utmost importance for wireless sensor networks deployed without any possibility of battery replenishments. Thus, design of energy efficient algorithms and protocols must consider resource constraints while maintaining the desired level of QoS.

In this paper, we present EE-MAC, an Energy Efficient medium access control (MAC) protocol for distributed wireless sensor networks. EE-MAC achieves a low-duty-cycle and hence low energy consumption through optimized sleep intervals while transitioning between sleep and active states. We consider a weighted linear combination of delay and energy saving as the performance metrics and through extensive simulations, we observe reduced energy consumption at the cost of increased delay. EE-MAC also improves the delay performance for fixed number of nodes compared to S-MAC.
\end{abstract}

\section{INTRODUCTION}

Wireless sensor networks (WSNs) are designed to sense and collect information from the environment. A WSN is composed of a large number of sensor nodes scattered over the region of interest. Sensor nodes are small and inexpensive devices, which have limited data processing capabilities, low transmission rates and small batteries. Sensor nodes are equipped with radio interfaces to perform their tasks such as data transmission to a common sink node [1], [2].

Due to the environmental constraints, it is generally not possible to replace or charge the batteries of sensor nodes after the network is deployed. Therefore, energy efficiency is critical to prolong the lifetime of a WSN. Techniques to optimize energy usage can be employed at various layers of the protocol stack [2]-[5]. In a distributed sensor network, the design of the MAC protocol is particularly important since it resolves channel contention among nodes and determines which node should access the shared channels and for how long. QoS provisioning poses additional challenges to the design of MAC protocols as guaranteeing delay requirements and sustaining bandwidth constraints can be compromised due to increased mutual access interference [6].

MAC protocols developed for WSNs can be broadly classified into two main categories: scheduling-based and contention-based. Each protocol is designed for specific topologies or applications [7]. Scheduling-based approaches form schedules, which allow each node in the network to access the channel and communicate with other nodes. In contention-based approaches, nodes compete for the wireless medium to acquire the access for data transmission. This work is motivated from the well-known MAC protocol S-MAC [2], where nodes sleep in a periodic manner to reduce energy consumption. As events being sensed could be sporadic, sensors do not sense at all times. Each node turns off its radio for a certain time and wakes up to check for receptions periodically. The listen and sleep states forms a frame. Though listening time is dictated by the limitations of MAC and PHY layers, there are no such restrictions for the sleep time. Thus, the duty cycle defined as the ratio of listen interval to frame duration is small for large sleep times and vice-versa. With events being sensed are sporadic, it is not necessary that the sleep times remain fixed. We argue that the sleep times should be optimized depending on the sensed activity.

In this paper, we propose EE-MAC, an Energy Efficient MAC layer protocol with variable sleep intervals for WSNs. We compute the duty cycle usage of EE-MAC and propose the selection of sleep intervals based on a 2-state Markov model [8]. We define the duty cycle as the fraction of time a node is active and use that to define the consumed energy and the incurred delay. As for the objective function, we propose a weighted linear combination of energy and delay after normalization. The objection function is then minimized to find the optimal value of the sleep times. Through exhaustive simulations, we show how EE-MAC performs with respect to $\mathrm{S}-\mathrm{MAC}$ in terms of energy consumption and delay.

\section{RELATED WORK}

There is a rich literature on energy efficient MAC protocols in WSNs [9]. The proposed protocols focus on reducing all sources of wasted energy such as idle listing or overhearing. The collisions also waste energy due to extra transmissions to handle the discarded packets. Control packet overhead can consume extra energy by the unnecessary transition unless designed according to the network requirements.

Ye et al. [2] proposed S-MAC, a contention-based MAC protocol for WSNs. S-MAC establishes low-duty-cycle operation to reduce energy consumption by periodically putting nodes into sleep and active states. Nodes coordinate their sleep schedules rather than having random sleep periods. QAMAC by Gao [10], which is based on S-MAC protocol, improves energy efficiency by coordinating the contention window dynamically. AsyMAC by Wang et al. [11], [12] is designed for wireless networks with asymmetric links. AsyMAC uses a set of concepts and metrics characterizing the ability of MAC to silence nodes which could cause collisions. Adaptive Coordinated Medium Access Control (AC-MAC) 


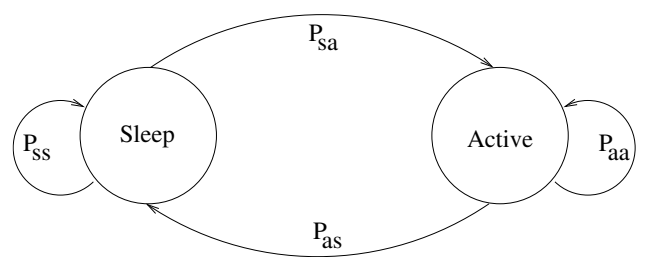

Fig. 1. 2-state (Active and Sleep) Markov model.

protocol proposed by Ai et al. [13] is a contention-based MAC protocol for WSNs. AC-MAC introduces adaptive duty cycle that depends on different traffic loads and provides optimized trade-off strategies for energy, throughput and latency. Aydin et al. [5]

Multi-token based MAC protocol with sleep scheduling for WSNs [14] by Dash et al. aims to improve energy efficiency along with faster data transmission, data aggregation, data accuracy and low latency in hop-by-hop delivery. The limitation of this protocol is the high latency for finding a new neighbor. E-BMA by Shafiullah et al. [15] is proposed to achieve energy efficiency for wireless data communication networks with low and medium traffic.

\section{EE-MAC PROTOCOL}

The main goal of EE-MAC is to reduce energy consumption and optimize delay performance. This goal is achieved by determining the optimal value of the sleep interval based on prevailing conditions.

\section{A. State Model}

The node activities in EE-MAC can be represented using the Gilbert-Elliott model [8], [16]. This 2-state Markov model is shown in Fig. 1, where transitions from 'sleep' state to 'active' state occurs with probability $P_{s a}$. Similarly, transitions from 'active' state to 'sleep' state occurs with probability $P_{a s}$. Transitions from each state to itself is also shown. Thus, the probability of being in the active state is

$$
P_{a}=P_{s a}+P_{a a} .
$$

Similarly, the probability of being in the sleep state is

$$
P_{s}=P_{a s}+P_{s s}
$$

It is to be noted that we do not treat receiving, transmitting, and listening as different states as they are included in the 'active' state.

It is assumed that the active times and sleep times are exponentially distributed. Let us define $\overline{t_{a}}$ as the average time a node spends in the active state. Similarly, $\overline{t_{s}}$ is the average time a node spends in the sleep state. Thus, we can define the duty cycle of the node as:

$$
\rho=\frac{\bar{t}_{a}}{\bar{t}_{a}+\bar{t}_{s}}
$$

i.e., the fraction of time the node is active. It can be noted that, $P_{a}=\rho$ and $P_{s}=(1-\rho)$.

\section{B. Energy and Delay}

Although it is desirable to have a low duty cycle, it compromises the delay performance. For instance, if a node sleeps while there is data transmission to it, the node will incur some delay in its response, which increases as the sleep times become longer. Thus, while optimizing the sleep intervals, the deterioration in the delay response must be taken into account.

Given different energy consumptions of two states, we define the total energy consumed per unit time per node, $E$, as follows:

$$
E=E_{\text {Active }}+E_{\text {Sleep }}
$$

where $E_{\text {Active }}$ is the average energy consumed per unit time in active state and $E_{\text {Sleep }}$ is the average energy consumed per unit time in sleep state. If $W_{a}$ and $W_{s}$ are the energy consumed per unit time during the active and sleep states respectively, then $E_{\text {Active }}=\rho W_{a}$ and $E_{\text {Sleep }}=(1-\rho) W_{s}$. Thus, the total consumed energy is defined as follows:

$$
E=\rho W_{a}+(1-\rho) W_{s}
$$

For a sleeping node, the expected time to wake up is $\bar{t}_{s}$, irrespective of the time it has been sleeping. This is a result of the assumption of exponential sleep time distribution, hence memoryless. Thus, delay can be defined as $D=\bar{t}_{s}$.

\section{Normalization of Energy and Delay}

To include both energy $E$ and delay $D$ in a combined metric, we must normalize them in a way so that they map to a number between 0 and 1 . If we assume $\max \left(W_{a}, W_{s}\right)=W_{a}$ as energy spent in active mode is more than the energy spent in the sleep mode, then the maximum value for $E$ is $W_{a}$. This happens when $\rho=1$, i.e., the node is always in the active state. Thus, we define the normalized energy, $E_{n o r m}$, as:

$$
E_{n o r m}=\frac{\rho W_{a}+(1-\rho) W_{s}}{W_{a}}
$$

Similarly, we seek a function for $D$ such that when $t_{s} \rightarrow 0$, $D \rightarrow 0$ and when $t_{s} \rightarrow \infty, D \rightarrow 1$. We define the normalized delay, $D_{\text {norm }}$, as $D_{\text {norm }}=1-\frac{1}{\bar{t}_{s}}$.

\section{Combined Metric}

We define the combined metric as a linear combination of $E_{\text {norm }}$ and $D_{\text {norm }}$ as:

$$
U=w_{1} \times E_{n o r m}+w_{2} \times D_{\text {norm }}
$$

where $w_{1}$ and $w_{2}$ are the corresponding weighing factors and $w_{1}+w_{2}=1$.

We seek to find the value of $t_{s}$ for which $U$ is minimized. Thus, we take partial derivatives and equate to 0 :

$$
\left[\frac{\partial U}{\partial t_{s}}\right]=\left[\frac{\partial E_{n o r m}}{\partial t_{s}}\right]+\left[\frac{\partial D_{\text {norm }}}{\partial t_{s}}\right]=0
$$

Solving equation (5), we get

$$
\bar{t}_{s}=\sqrt{\frac{w_{1} W_{a} \bar{t}_{a}-w_{2} W_{a}}{W_{s} w_{1} \bar{t}_{a}}}
$$

For $\bar{t}_{s}$ to have a real value, $w_{1} \bar{t}_{a} \geq w_{2}$. 


\section{Simulation Model and Results}

We evaluate EE-MAC and compare it with S-MAC in terms of energy consumption and delay. In the simulations, 700 nodes are scattered over a square area, where they remain active for a certain duration $\overline{t_{a}}$. The sleep times are varied as per exponential distribution with a mean $\overline{t_{s}}$.

We simulate for both fixed and varying $t_{s}$ values. Although the sleep times are exponentially distributed in theory, there is an upper bound $d_{\max }$ on the time a node can sleep after which it has to wake up irrespective of any triggers in real-life applications. For the combined metric, we use $w_{1}=w_{2}=0.5$, i.e., both energy and delay are equally important. As for the energy consumption in active and sleep states, we assume $W_{a}=36$ and $W_{s}=0.015$ as specified in [2]. Table I summarizes the simulation parameters.

TABLE I

SIMULATION PARAMETERS

\begin{tabular}{|c|c|}
\hline number of nodes & $100-700$ \\
\hline$W_{a}$ & 36 \\
\hline$W_{s}$ & 0.015 \\
\hline$w_{1}$ & $0.5 ; 0.1$ \\
\hline$w_{2}$ & $0.5 ; 0.9$ \\
\hline
\end{tabular}

The performance of the proposed protocol is presented in Figures 2-4. In Fig. 2, we show how the energy consumption varies with increasing sleep times for a fixed active time $\left(t_{a}=100,200\right.$, and 300). As expected, the more a node sleeps the less would be the energy consumption. Additionally, with lower active times, energy consumption is also reduced. As shown in Fig. 3, the savings in energy due to increased sleep times is offset by the delay degradations. We used two different values for the maximum delay allowed for a node to sleep i.e., $d_{\max }=300$ and $d_{\max }=400$. In Fig. 4, the combined utility is given for $t_{a}=100,200$, and 300 .

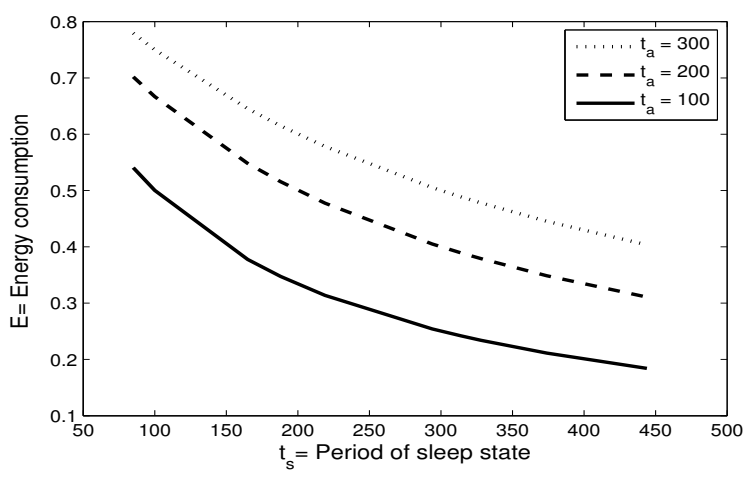

Fig. 2. Energy consumption vs. sleep times.

In Figures 5-8, we compare the performance of EE-MAC with S-MAC. Fig. 5 illustrates the energy consumption for EEMAC and S-MAC for nodes 100 to 700 with $w_{1}=w_{2}=0.5$ (same weights for energy and delay). We can see that EE-MAC performs better in energy consumption for smaller number of nodes. However, as the number of nodes increase, the energy

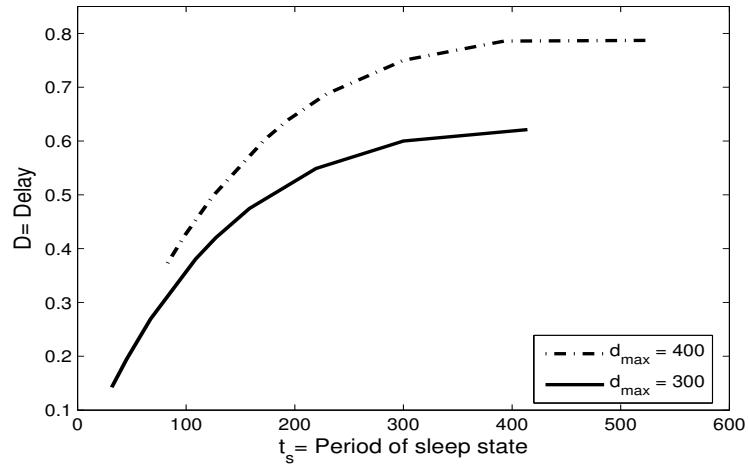

Fig. 3. Delay vs. sleep times.

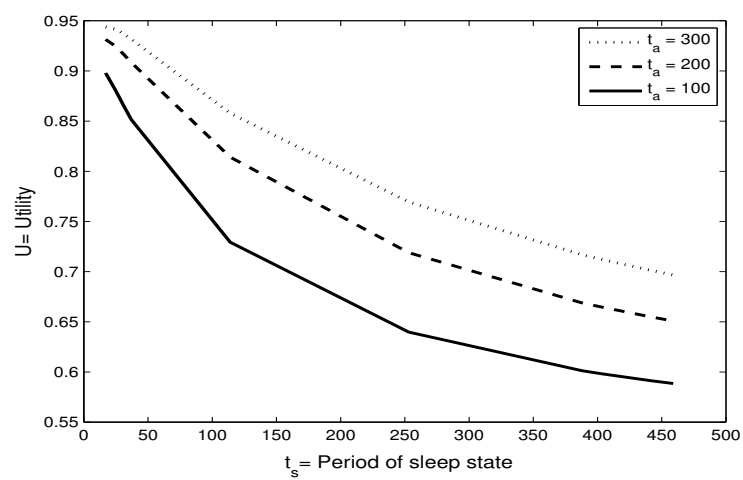

Fig. 4. Combined utility when $t_{a}=100, t_{a}=200, t_{a}=300$.

savings of EE-MAC also increases accordingly. In Fig. 6, we set $w_{1}=0.9$ and $w_{2}=0.1$ to show the effect of varied importance of delay and energy. The results show that the energy consumption in EE-MAC with the new weight values is also less than the energy consumption in S-MAC.

Fig. 7 shows the performances of EE-MAC and S-MAC in terms of delay, for a fixed number of nodes and $t_{s}=100$. With high sleep times, EE-MAC performs better as S-MAC is expected to have an inefficient delay performance. The delay performances improve when the average sleep time is

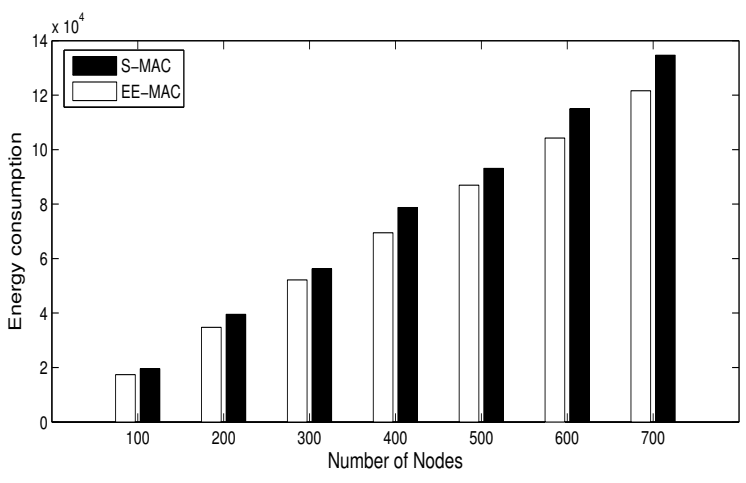

Fig. 5. Energy consumption for $w_{1}=w_{2}=0.5$ 




Fig. 6. Energy Consumption for $w_{1}=0.9$ and $w_{2}=0.1$

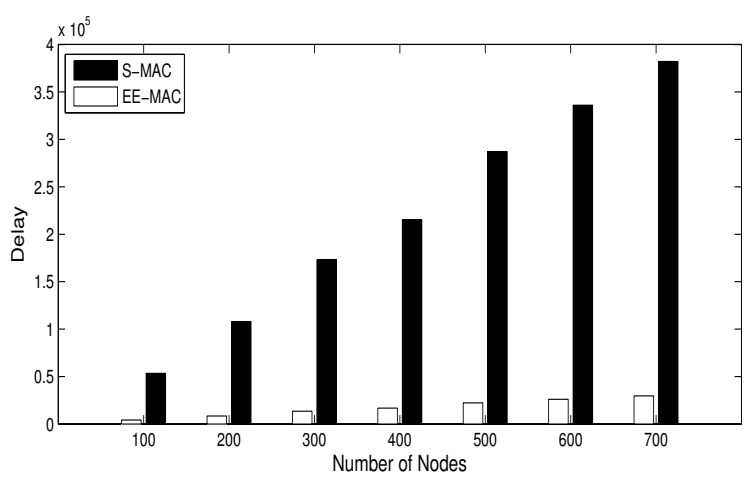

Fig. 7. Delay with $t_{s}=100$

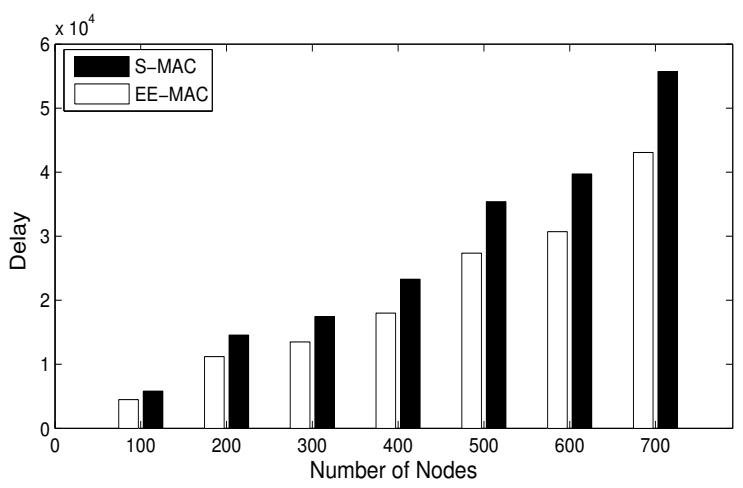

Fig. 8. Delay with $t_{s}=20$

reduced. Fig. 8 presents the delay performances for $t_{s}=20$. Further reduction of $t_{s}$ shows better delay performance for EE-MAC than S-MAC, but with compromised energy savings. Fig. 7 and Fig. 8 illustrate that the delay performance of EEMAC is better than S-MAC for variable sleep times. The results reveal that it is best to have variable sleep times that can be tuned based on the sensing activity and the desired tradeoff between energy and delay. The performance difference between the protocols is more significant for delay than the energy consumption.

\section{CONCLusions}

Achieving energy efficiency in WSNs is of utmost importance. Since sensor nodes consume more power while sensing and transmitting compared to idle time, achieving a low duty cycle improves the performance in terms of energy consumption. We achieve this goal by putting nodes to sleep at the cost of degraded delay performance. To that end, we propose Energy Efficient MAC layer protocol, called EE$\mathrm{MAC}$, and derive the energy consumption and the incurred delay when the node switches between the two states. We also propose a combined metric which is a linear sum of the two and find the optimal sleep time. Through extensive simulation experiments, we observe the performance improvement of EEMAC compared to S-MAC.

\section{REFERENCES}

[1] D. Bruneo, A. Puliafito, and M. Scarpa, "Dependability analysis of wireless sensor networks with active-sleep cycles and redundant nodes," in Workshop on DYnamic Aspects in DEpendability Models for FaultTolerant Systems, pp. 25-30, Apr. 2010.

[2] W. Ye, J. Heidemann, and D. Estrin, "Medium access control with coordinated adaptive sleeping for wireless sensor networks," IEEE/ACM Transactions on Networking, vol. 12, pp. 493-506, June 2004.

[3] M. I. Akbaş and D. Turgut, "Lightweight Routing with QoS Support in Wireless Sensor and Actor Networks," in IEEE GLOBECOM, pp. 1-5, Dec. 2010.

[4] M. Di Francesco, G. Anastasi, M. Conti, S. Das, and V. Neri, "Reliability and Energy-Efficiency in IEEE 802.15.4/ZigBee Sensor Networks:An Adaptive and Cross-Layer Approach," IEEE Journal on Selected Areas on Communications (JSAC), vol. 29, pp. 1508 -1524, Sept. 2011.

[5] N. Aydin, S. H. You, B. Turgut, and D. Turgut, "Scenario-based performance analysis of MAC protocols for wireless sensor networks," in International Conference on Wireless Networks (ICWN), pp. 105-111, June 2005.

[6] S. De, C. Qiao, D. Pados, M. Chatterjee, and S. Philip, "An integrated cross-layer study of wireless CDMA sensor networks," IEEE Journal on Selected Areas on Communications (JSAC), Special Issue on Quality of Service Delivery in Variable Topology Networks, vol. 22, pp. 193-205, Sept 2004.

[7] P. Ji, C. Wu, Y. Zhang, and Z. ha, "Research of an energy-aware MAC protocol in Wireless Sensor Network," in Control and Decision Conference, pp. 4686-4690, July 2008.

[8] E. N. Gilbert, "Capacity of a burst-noise channel," Bell System Technical Journal, vol. 39, pp. 1253-1265, Sept. 1960.

[9] P. Huang, L. Xiao, S. Soltani, M. Mutka, and N. Xi, "The evolution of MAC protocols in wireless sensor networks: A survey," IEEE Cоттиnications Surveys Tutorials, vol. 15, pp. 101-120, Feb. 2013.

[10] L. Gao, "A Energy Consumption Improvements of S-MAC in WSN," in International Conference on Internet Technology and Applications (iTAP), pp. 1-3, Aug. 2011.

[11] G. Wang, D. Turgut, L. Bölöni, Y. Ji, and D. C. Marinescu, "A MAC layer protocol for wireless networks with asymmetric links," Ad Hoc Networks, vol. 6, pp. 424-440, May 2008.

[12] G. Wang, D. Turgut, L. Bölöni, Y. Ji, and D. C. Marinescu, "A simulation study of a MAC layer protocol for wireless networks with asymmetric links," in International Conference on Wireless Communications and Mobile Computing (IWCMC), pp. 929-936, July 2006.

[13] J. Ai, J. Kong, and D. Turgut, "An adaptive coordinated medium access control for wireless sensor networks," in International Symposium on Computers and Communications, pp. 214-219, June 2004.

[14] S. Dash, A. R. Swain, and A. Ajay, "Reliable Energy Aware Multitoken Based MAC Protocol for WSN," in IEEE AINA, pp. 144-151, Mar. 2012.

[15] G. M. Shafiullah, S. A. Azad, and A. Ali, "Energy-efficient wireless MAC protocols for railway monitoring applications," IEEE Transactions on Intelligent Transportation Systems, vol. 14, pp. 649-659, June 2013.

[16] E. O. Elliott, "Estimates of Error Rates for Codes on Burst-Noise Channels," Bell System Technical Journal, vol. 42, pp. 1977-1997, Sept. 1963. 